Acta Crystallographica Section E

Structure Reports

Online

ISSN 1600-5368

\section{1,1,1-Trichloro-2,2-bis(4-iodophenyl)- ethane}

\section{Graham Smith}

Science and Engineering Faculty, Queensland University of Technology, GPO Box 2434, Brisbane, Queensland 4001, Australia

Correspondence e-mail: g.smith@qut.edu.au

Received 10 July 2012; accepted 16 July 2012

Key indicators: single-crystal X-ray study; $T=200 \mathrm{~K}$; mean $\sigma(\mathrm{C}-\mathrm{C})=0.013 \AA$; $R$ factor $=0.044 ; w R$ factor $=0.112 ;$ data-to-parameter ratio $=16.9$.

In the structure of the title compound, $\mathrm{C}_{14} \mathrm{H}_{9} \mathrm{Cl}_{3} \mathrm{I}_{2}$, which is the 4-iodophenyl analogue of the insecticide DDT [1,1,1-trichloro-2,2-bis(4-chlorophenyl)ethane], isomorphism between the two compounds has been confirmed. In the molecule, the dihedral angle between the planes of the two benzene rings is $65.8(4)^{\circ}$ which compares with $64.7(7)^{\circ}$ in DDT.

\section{Related literature}

For the determination of crystal data for the title compound and the $p$-bromo substituted DDT analogue, see: Schneider \& Fankuchen (1946). For the structures of DDT and related analogues, see: DeLacy \& Kennard (1972); Hovmöller et al. (1978).<smiles>ClC(Cl)(Cl)C(c1ccc(I)cc1)c1ccc(I)cc1</smiles>

\section{Experimental}

Crystal data

$\begin{array}{ll}\mathrm{C}_{14} \mathrm{H}_{9} \mathrm{Cl}_{3} \mathrm{I}_{2} & V=1606.61(7) \AA^{3} \\ M_{r}=537.36 & Z=4 \\ \text { Orthorhombic, } P c a 2_{1} & \text { Mo Ko radiation } \\ a=9.8117(3) \AA & \mu=4.40 \mathrm{~mm}^{-1} \\ b=20.3445(4) \AA & T=200 \mathrm{~K} \\ c=8.0486(2) \AA & 0.25 \times 0.20 \times 0.08 \mathrm{~mm}\end{array}$

\section{Data collection}

Oxford Diffraction Gemini-S CCDdetector diffractometer

Absorption correction: multi-scan

(CrysAlis PRO; Agilent, 2012)

$T_{\min }=0.386, T_{\max }=0.980$

Refinement

$R\left[F^{2}>2 \sigma\left(F^{2}\right)\right]=0.044$

$w R\left(F^{2}\right)=0.112$

$S=1.08$

2905 reflections

172 parameters

1 restraint

5183 measured reflections 2905 independent reflections 2687 reflections with $I>2 \sigma(I)$ $R_{\text {int }}=0.028$

Data collection: CrysAlis PRO (Agilent, 2012); cell refinement: CrysAlis PRO; data reduction: CrysAlis PRO; program(s) used to solve structure: SIR92 (Altomare et al., 1993); program(s) used to refine structure: SHELXL97 (Sheldrick, 2008) within WinGX (Farrugia, 1999); molecular graphics: PLATON (Spek, 2009); software used to prepare material for publication: PLATON.

The author acknowledges financial support from the Australian Research Council and the Science and Engineering Faculty and the University Library, Queensland University of Technology.

Supplementary data and figures for this paper are available from the IUCr electronic archives (Reference: SU2477).

\section{References}

Agilent (2012). CrysAlis PRO. Agilent Technologies Ltd, Yarnton, England. Altomare, A., Cascarano, G., Giacovazzo, C. \& Guagliardi, A. (1993). J. Appl. Cryst. 26, 343-350.

DeLacy, T. P. \& Kennard, C. H. L. (1972). J. Chem. Soc. Perkin Trans. 2, pp. $2148-2152$.

Farrugia, L. J. (1999). J. Appl. Cryst. 32, 837-838.

Flack, H. D. (1983). Acta Cryst. A39, 876-881.

Hovmöller, S., Smith, G. \& Kennard, C. H. L. (1978). Acta Cryst. B34, 30163021.

Schneider, M. \& Fankuchen, I. (1946). J. Am. Chem. Soc. 68, 2669-2670.

Sheldrick, G. M. (2008). Acta Cryst. A64, 112-122.

Spek, A. L. (2009). Acta Cryst. D65, 148-155. 


\section{supporting information}

Acta Cryst. (2012). E68, o2504 [https://doi.org/10.1107/S1600536812032254]

\section{1,1,1-Trichloro-2,2-bis(4-iodophenyl)ethane}

\section{Graham Smith}

\section{S1. Comment}

The title compound is the $p$-iodophenyl analogue of the insecticide DDT [1,1,1-trichloro-2,2-bis(4-chlorophenyl)ethane] which with the $p$-bromophenyl analogue provided crystal data (Schneider \& Fankuchen, 1946) that indicated a probable isomorphous series [orthorhombic, space group $P c a 2_{1}, Z=4$ : for the $p$-Cl analogue (DDT), $a=9.963$ (1), $b=19.200$ (2), $c=7.887$ (1) $\AA, V=1509.0 \AA^{3}$ [from the crystal structure of DDT (DeLacy \& Kennard, 1972); for the 4-bromophenyl analogue, $\left.a=9.93, b=19.68, c=7.93 \AA, V=1549 \AA^{3}\right]$. The structure of the title compound, for which the crystal data was also reported by Schneider \& Fankuchen (1946), is reported herein.

With the title compound (Fig. 1), isomorphism with DDT as suggested from the crystal data has been confirmed on the basis of space group, cell parameters and the molecular structures. The dihedral angle between the two phenyl planes in this compound $\left[65.8(4)^{\circ}\right]$ compares with $64.7(7)^{\circ}$ in the structure of DDT (DeLacy \& Kennard, 1972). Stabilizing the ring conformation is an intramolecular aromatic $\mathrm{C} 6 A-\mathrm{H} \cdots \mathrm{Cl} 2$ interaction $[\mathrm{D} \cdots \mathrm{A}=3.335(10) \AA]$.

In the crystal, there are relatively short I $4 A \cdots \mathrm{Cl} 1$ and I $4 A \cdots \mathrm{I} 4 A$ contacts [3.777 (2) and 4.1502 (9) $\AA$, respectively] but otherwise no other significant intermolecular interactions are present (Fig. 2).

\section{S2. Experimental}

The title compound was obtained as an analytical reference standard from the U.S.Public Health Service. The original crystal data was reported by Schneider \& Fankuchen (1946). Small colourless plate-like crystals of the title compound, suitable for X-ray analysis, were obtained by room temperature evaporation of a solution in isopropyl alcohol.

\section{S3. Refinement}

Hydrogen atoms were included in the refinement at calculated positions $[\mathrm{C}-\mathrm{H}=0.93 \AA$ (aromatic) or $0.98 \AA$ (methine), with $U_{\text {iso }}(\mathrm{H})=1.2 U_{\text {eq }}(\mathrm{C})$, using a riding-model approximation. The maximum difference electron density peak was 0.80 $\mathrm{e} \AA^{-3}$, adjacent to atom I4A. 


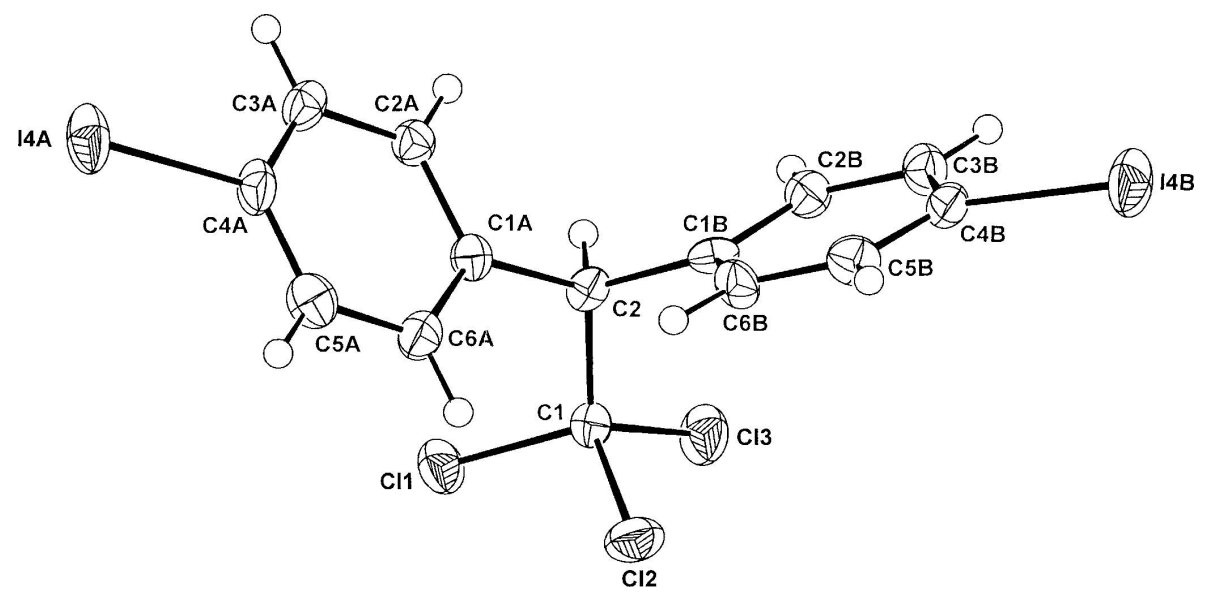

Figure 1

Molecular conformation and atom numbering scheme for the title molecule, with displacement ellipsoids drawn at the $50 \%$ probability level.

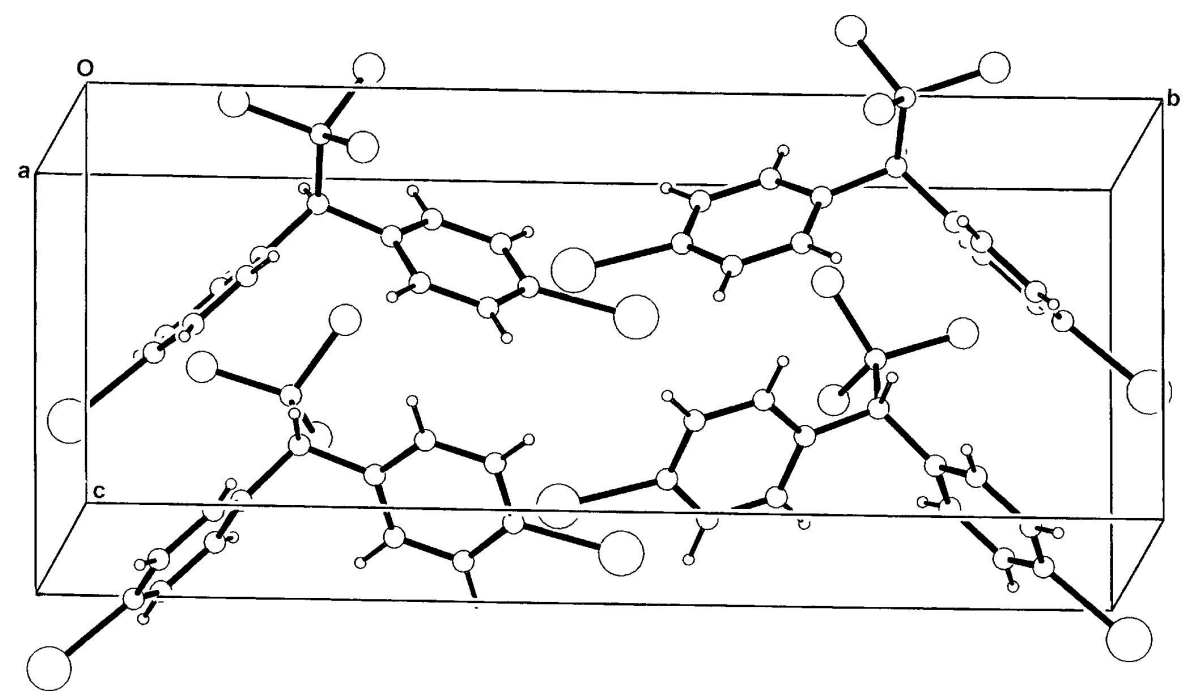

Figure 2

A perspective view of the crystal packing of the title compound viewed along the $a$ axis.

\section{1,1,1-trichloro-2,2-bis(4-iodophenyl)ethane}

\section{Crystal data}

$\mathrm{C}_{14} \mathrm{H}_{9} \mathrm{Cl}_{3} \mathrm{I}_{2}$

$M_{r}=537.36$

Orthorhombic, $P c a 2_{1}$

Hall symbol: P 2c -2ac

$a=9.8117(3) \AA$

$b=20.3445(4) \AA$

$c=8.0486(2) \AA$

$V=1606.61(7) \AA^{3}$

$Z=4$
$F(000)=1000$

$D_{\mathrm{x}}=2.222 \mathrm{Mg} \mathrm{m}^{-3}$

Mo $K \alpha$ radiation, $\lambda=0.71073 \AA$

Cell parameters from 2427 reflections

$\theta=3.2-28.7^{\circ}$

$\mu=4.40 \mathrm{~mm}^{-1}$

$T=200 \mathrm{~K}$

Plate, colourless

$0.25 \times 0.20 \times 0.08 \mathrm{~mm}$ 


\section{Data collection}

Oxford Diffraction Gemini-S CCD-detector diffractometer

Radiation source: Enhance (Mo) X-ray source Graphite monochromator

Detector resolution: 16.077 pixels $\mathrm{mm}^{-1}$

$\omega$ scans

Absorption correction: multi-scan

(CrysAlis PRO; Agilent, 2012)

$T_{\min }=0.386, T_{\max }=0.980$

Refinement

Refinement on $F^{2}$

Least-squares matrix: full

$R\left[F^{2}>2 \sigma\left(F^{2}\right)\right]=0.044$

$w R\left(F^{2}\right)=0.112$

$S=1.08$

2905 reflections

172 parameters

1 restraint

Primary atom site location: structure-invariant direct methods

Secondary atom site location: difference Fourier map

Special details

Geometry. Bond distances, angles etc. have been calculated using the rounded fractional coordinates. All su's are estimated from the variances of the (full) variance-covariance matrix. The cell e.s.d.'s are taken into account in the estimation of distances, angles and torsion angles

Refinement. Refinement of $F^{2}$ against ALL reflections. The weighted $R$-factor $w R$ and goodness of fit $S$ are based on $F^{2}$, conventional $R$-factors $R$ are based on $F$, with $F$ set to zero for negative $F^{2}$. The threshold expression of $F^{2}>\sigma\left(F^{2}\right)$ is used only for calculating $R$-factors $(\mathrm{gt}) \mathrm{etc}$. and is not relevant to the choice of reflections for refinement. $R$-factors based on $F^{2}$ are statistically about twice as large as those based on $F$, and $R$ - factors based on ALL data will be even larger.

Fractional atomic coordinates and isotropic or equivalent isotropic displacement parameters $\left(\AA^{2}\right)$

\begin{tabular}{lllll}
\hline & $x$ & $y$ & $z$ & $U_{\text {iso }} * / U_{\text {eq }}$ \\
\hline I4A & $0.95323(7)$ & $0.01062(3)$ & $1.18735(8)$ & $0.0400(2)$ \\
I4B & $0.89471(7)$ & $0.54100(3)$ & $0.90275(11)$ & $0.0422(2)$ \\
C11 & $1.0549(2)$ & $0.15878(11)$ & $0.4415(3)$ & $0.0316(7)$ \\
C12 & $1.1944(2)$ & $0.27227(12)$ & $0.5751(3)$ & $0.0359(7)$ \\
C13 & $0.9773(3)$ & $0.28835(12)$ & $0.3400(3)$ & $0.0366(7)$ \\
C1 & $1.0324(8)$ & $0.2408(4)$ & $0.5112(12)$ & $0.026(3)$ \\
C1A & $0.9376(8)$ & $0.1901(4)$ & $0.7842(11)$ & $0.022(3)$ \\
C1B & $0.9112(8)$ & $0.3126(4)$ & $0.7238(11)$ & $0.023(3)$ \\
C2 & $0.9238(8)$ & $0.2436(4)$ & $0.6522(10)$ & $0.020(3)$ \\
C2A & $0.8197(9)$ & $0.1581(4)$ & $0.8344(11)$ & $0.024(2)$ \\
C2B & $0.9956(8)$ & $0.3350(4)$ & $0.8512(12)$ & $0.027(3)$ \\
C3A & $0.8211(9)$ & $0.1073(4)$ & $0.9512(11)$ & $0.027(3)$ \\
C3B & $0.9885(8)$ & $0.3989(4)$ & $0.9066(15)$ & $0.031(3)$ \\
C4A & $0.9450(10)$ & $0.0899(4)$ & $1.0187(13)$ & $0.030(3)$ \\
C4B & $0.8945(9)$ & $0.4409(5)$ & $0.8347(13)$ & $0.029(3)$
\end{tabular}

5183 measured reflections

2905 independent reflections

2687 reflections with $I>2 \sigma(I)$

$R_{\text {int }}=0.028$

$\theta_{\max }=26.0^{\circ}, \theta_{\min }=3.4^{\circ}$

$h=-10 \rightarrow 12$

Hydrogen site location: inferred from

neighbouring sites

$\mathrm{H}$-atom parameters constrained

$w=1 /\left[\sigma^{2}\left(F_{\mathrm{o}}^{2}\right)+(0.0574 P)^{2}+5.2653 P\right]$

where $P=\left(F_{\mathrm{o}}^{2}+2 F_{\mathrm{c}}{ }^{2}\right) / 3$

$(\Delta / \sigma)_{\max }=0.001$

$\Delta \rho_{\max }=0.80 \mathrm{e} \AA^{-3}$ pairs

Absolute structure parameter: -0.02 (4) 


$\begin{array}{lllll}\text { C5A } & 1.0643(9) & 0.1209(5) & 0.9744(13) & 0.032(3) \\ \text { C5B } & 0.8044(8) & 0.4195(4) & 0.7141(12) & 0.029(3) \\ \text { C6A } & 1.0600(9) & 0.1711(5) & 0.8569(13) & 0.029(3) \\ \text { C6B } & 0.8130(8) & 0.3553(4) & 0.6603(12) & 0.027(3) \\ \text { H2 } & 0.83640 & 0.23500 & 0.59740 & 0.0250^{*} \\ \text { H2A } & 0.73680 & 0.17080 & 0.78850 & 0.0290^{*} \\ \text { H2B } & 1.05770 & 0.30630 & 0.89950 & 0.0320^{*} \\ \text { H3A } & 0.74120 & 0.08600 & 0.98230 & 0.0320^{*} \\ \text { H3B } & 1.04570 & 0.41360 & 0.99090 & 0.0360^{*} \\ \text { H5A } & 1.14670 & 0.10850 & 1.02230 & 0.0380^{*} \\ \text { H5B } & 0.73940 & 0.44780 & 0.67010 & 0.0350^{*} \\ \text { H6A } & 1.14030 & 0.19220 & 0.82650 & 0.0350^{*} \\ \text { H6B } & 0.75230 & 0.34020 & 0.58020 & 0.0320^{*}\end{array}$

Atomic displacement parameters $\left(\AA^{2}\right)$

\begin{tabular}{lllllll}
\hline & $U^{11}$ & $U^{22}$ & $U^{33}$ & $U^{12}$ & $U^{13}$ & $U^{23}$ \\
\hline I4A & $0.0686(4)$ & $0.0249(3)$ & $0.0266(3)$ & $0.0085(3)$ & $0.0032(3)$ & $0.0055(3)$ \\
I4B & $0.0629(4)$ & $0.0206(3)$ & $0.0430(4)$ & $-0.0046(3)$ & $0.0038(4)$ & $-0.0056(3)$ \\
C11 & $0.0378(11)$ & $0.0244(11)$ & $0.0326(13)$ & $0.0046(8)$ & $0.0019(9)$ & $-0.0051(9)$ \\
C12 & $0.0264(10)$ & $0.0380(12)$ & $0.0433(15)$ & $-0.0087(9)$ & $0.0074(10)$ & $-0.0060(11)$ \\
C13 & $0.0510(13)$ & $0.0299(12)$ & $0.0290(12)$ & $0.0033(10)$ & $0.0040(11)$ & $0.0078(10)$ \\
C1 & $0.025(4)$ & $0.019(4)$ & $0.034(5)$ & $-0.001(3)$ & $0.003(4)$ & $-0.001(4)$ \\
C1A & $0.026(4)$ & $0.020(4)$ & $0.021(5)$ & $0.000(3)$ & $-0.002(3)$ & $-0.002(3)$ \\
C1B & $0.019(3)$ & $0.031(5)$ & $0.018(5)$ & $-0.003(3)$ & $0.004(3)$ & $0.000(3)$ \\
C2 & $0.018(4)$ & $0.022(4)$ & $0.021(5)$ & $-0.004(3)$ & $-0.007(3)$ & $0.003(3)$ \\
C2A & $0.024(4)$ & $0.022(4)$ & $0.026(4)$ & $-0.001(3)$ & $-0.001(4)$ & $-0.001(3)$ \\
C2B & $0.020(4)$ & $0.025(4)$ & $0.035(5)$ & $0.005(3)$ & $-0.008(4)$ & $0.002(4)$ \\
C3A & $0.035(4)$ & $0.021(4)$ & $0.024(5)$ & $-0.007(3)$ & $0.004(4)$ & $-0.003(3)$ \\
C3B & $0.027(4)$ & $0.034(5)$ & $0.031(5)$ & $-0.001(3)$ & $-0.003(4)$ & $-0.002(5)$ \\
C4A & $0.044(5)$ & $0.020(4)$ & $0.026(5)$ & $0.003(4)$ & $0.004(4)$ & $0.006(4)$ \\
C4B & $0.035(5)$ & $0.020(4)$ & $0.031(5)$ & $-0.002(4)$ & $0.012(4)$ & $0.001(4)$ \\
C5A & $0.033(5)$ & $0.032(5)$ & $0.030(5)$ & $0.005(4)$ & $-0.005(4)$ & $0.002(4)$ \\
C5B & $0.032(4)$ & $0.024(4)$ & $0.030(6)$ & $0.003(3)$ & $-0.001(4)$ & $0.004(4)$ \\
C6A & $0.028(4)$ & $0.024(4)$ & $0.035(6)$ & $-0.003(4)$ & $0.000(4)$ & $0.003(4)$ \\
C6B & $0.025(4)$ & $0.024(4)$ & $0.031(6)$ & $-0.002(3)$ & $-0.001(4)$ & $-0.002(4)$ \\
& & & & & & \\
\hline
\end{tabular}

Geometric parameters $\left(\AA,{ }^{\circ}\right)$

\begin{tabular}{llll}
\hline $\mathrm{I} 4 \mathrm{~A}-\mathrm{C} 4 \mathrm{~A}$ & $2.110(9)$ & $\mathrm{C} 3 \mathrm{~B}-\mathrm{C} 4 \mathrm{~B}$ & $1.384(13)$ \\
$\mathrm{I} 4 \mathrm{~B}-\mathrm{C} 4 \mathrm{~B}$ & $2.109(10)$ & $\mathrm{C} 4 \mathrm{~A}-\mathrm{C} 5 \mathrm{~A}$ & $1.377(13)$ \\
$\mathrm{C} 11-\mathrm{C} 1$ & $1.774(9)$ & $\mathrm{C} 4 \mathrm{~B}-\mathrm{C} 5 \mathrm{~B}$ & $1.383(13)$ \\
$\mathrm{C} 12-\mathrm{C} 1$ & $1.789(8)$ & $\mathrm{C} 5 \mathrm{~A}-\mathrm{C} 6 \mathrm{~A}$ & $1.393(15)$ \\
$\mathrm{C} 13-\mathrm{C} 1$ & $1.768(9)$ & $\mathrm{C} 5 \mathrm{~B}-\mathrm{C} 6 \mathrm{~B}$ & $1.379(12)$ \\
$\mathrm{C} 1-\mathrm{C} 2$ & $1.558(12)$ & $\mathrm{C} 2-\mathrm{H} 2$ & 0.9800 \\
$\mathrm{C} 1 \mathrm{~A}-\mathrm{C} 2$ & $1.527(12)$ & $\mathrm{C} 2 \mathrm{~A}-\mathrm{H} 2 \mathrm{~A}$ & 0.9300 \\
$\mathrm{C} 1 \mathrm{~A}-\mathrm{C} 2 \mathrm{~A}$ & $1.388(12)$ & $\mathrm{C} 2 \mathrm{~B}-\mathrm{H} 2 \mathrm{~B}$ & 0.9300 \\
$\mathrm{C} 1 \mathrm{~A}-\mathrm{C} 6 \mathrm{~A}$ & $1.391(12)$ & $\mathrm{C} 3 \mathrm{~A}-\mathrm{H} 3 \mathrm{~A}$ & 0.9300
\end{tabular}




\begin{tabular}{|c|c|c|c|}
\hline $\mathrm{C} 1 \mathrm{~B}-\mathrm{C} 2$ & $1.523(12)$ & $\mathrm{C} 3 \mathrm{~B}-\mathrm{H} 3 \mathrm{~B}$ & 0.9300 \\
\hline $\mathrm{C} 1 \mathrm{~B}-\mathrm{C} 2 \mathrm{~B}$ & $1.395(12)$ & $\mathrm{C} 5 \mathrm{~A}-\mathrm{H} 5 \mathrm{~A}$ & 0.9300 \\
\hline $\mathrm{C} 1 \mathrm{~B}-\mathrm{C} 6 \mathrm{~B}$ & $1.394(12)$ & $\mathrm{C} 5 \mathrm{~B}-\mathrm{H} 5 \mathrm{~B}$ & 0.9300 \\
\hline $\mathrm{C} 2 \mathrm{~A}-\mathrm{C} 3 \mathrm{~A}$ & $1.397(12)$ & C6A-H6A & 0.9300 \\
\hline $\mathrm{C} 2 \mathrm{~B}-\mathrm{C} 3 \mathrm{~B}$ & $1.376(12)$ & $\mathrm{C} 6 \mathrm{~B}-\mathrm{H} 6 \mathrm{~B}$ & 0.9300 \\
\hline $\mathrm{C} 3 \mathrm{~A}-\mathrm{C} 4 \mathrm{~A}$ & $1.378(13)$ & & \\
\hline $\mathrm{C} 11-\mathrm{C} 1-\mathrm{Cl} 2$ & $108.5(4)$ & $\mathrm{C} 3 \mathrm{~B}-\mathrm{C} 4 \mathrm{~B}-\mathrm{C} 5 \mathrm{~B}$ & $121.7(9)$ \\
\hline $\mathrm{C} 11-\mathrm{C} 1-\mathrm{Cl} 3$ & $107.8(5)$ & $\mathrm{C} 4 \mathrm{~A}-\mathrm{C} 5 \mathrm{~A}-\mathrm{C} 6 \mathrm{~A}$ & $119.1(9)$ \\
\hline $\mathrm{C} 11-\mathrm{C} 1-\mathrm{C} 2$ & $110.5(6)$ & $\mathrm{C} 4 \mathrm{~B}-\mathrm{C} 5 \mathrm{~B}-\mathrm{C} 6 \mathrm{~B}$ & $118.7(8)$ \\
\hline $\mathrm{Cl} 2-\mathrm{C} 1-\mathrm{Cl} 3$ & $107.5(5)$ & $\mathrm{C} 1 \mathrm{~A}-\mathrm{C} 6 \mathrm{~A}-\mathrm{C} 5 \mathrm{~A}$ & $121.1(8)$ \\
\hline $\mathrm{C} 12-\mathrm{C} 1-\mathrm{C} 2$ & $112.7(6)$ & $\mathrm{C} 1 \mathrm{~B}-\mathrm{C} 6 \mathrm{~B}-\mathrm{C} 5 \mathrm{~B}$ & $121.2(8)$ \\
\hline $\mathrm{C} 13-\mathrm{C} 1-\mathrm{C} 2$ & $109.8(5)$ & $\mathrm{C} 1-\mathrm{C} 2-\mathrm{H} 2$ & 105.00 \\
\hline $\mathrm{C} 2-\mathrm{C} 1 \mathrm{~A}-\mathrm{C} 2 \mathrm{~A}$ & $117.6(7)$ & $\mathrm{C} 1 \mathrm{~A}-\mathrm{C} 2-\mathrm{H} 2$ & 105.00 \\
\hline $\mathrm{C} 2-\mathrm{C} 1 \mathrm{~A}-\mathrm{C} 6 \mathrm{~A}$ & $124.6(8)$ & $\mathrm{C} 1 \mathrm{~B}-\mathrm{C} 2-\mathrm{H} 2$ & 105.00 \\
\hline $\mathrm{C} 2 \mathrm{~A}-\mathrm{C} 1 \mathrm{~A}-\mathrm{C} 6 \mathrm{~A}$ & $117.8(8)$ & $\mathrm{C} 1 \mathrm{~A}-\mathrm{C} 2 \mathrm{~A}-\mathrm{H} 2 \mathrm{~A}$ & 119.00 \\
\hline $\mathrm{C} 2-\mathrm{C} 1 \mathrm{~B}-\mathrm{C} 2 \mathrm{~B}$ & $122.1(7)$ & $\mathrm{C} 3 \mathrm{~A}-\mathrm{C} 2 \mathrm{~A}-\mathrm{H} 2 \mathrm{~A}$ & 119.00 \\
\hline $\mathrm{C} 2-\mathrm{C} 1 \mathrm{~B}-\mathrm{C} 6 \mathrm{~B}$ & $119.5(7)$ & $\mathrm{C} 1 \mathrm{~B}-\mathrm{C} 2 \mathrm{~B}-\mathrm{H} 2 \mathrm{~B}$ & 119.00 \\
\hline $\mathrm{C} 2 \mathrm{~B}-\mathrm{C} 1 \mathrm{~B}-\mathrm{C} 6 \mathrm{~B}$ & $118.4(8)$ & $\mathrm{C} 3 \mathrm{~B}-\mathrm{C} 2 \mathrm{~B}-\mathrm{H} 2 \mathrm{~B}$ & 119.00 \\
\hline $\mathrm{C} 1-\mathrm{C} 2-\mathrm{C} 1 \mathrm{~A}$ & $114.8(7)$ & $\mathrm{C} 2 \mathrm{~A}-\mathrm{C} 3 \mathrm{~A}-\mathrm{H} 3 \mathrm{~A}$ & 121.00 \\
\hline $\mathrm{C} 1-\mathrm{C} 2-\mathrm{C} 1 \mathrm{~B}$ & $111.4(7)$ & $\mathrm{C} 4 \mathrm{~A}-\mathrm{C} 3 \mathrm{~A}-\mathrm{H} 3 \mathrm{~A}$ & 121.00 \\
\hline $\mathrm{C} 1 \mathrm{~A}-\mathrm{C} 2-\mathrm{C} 1 \mathrm{~B}$ & $113.6(7)$ & $\mathrm{C} 2 \mathrm{~B}-\mathrm{C} 3 \mathrm{~B}-\mathrm{H} 3 \mathrm{~B}$ & 121.00 \\
\hline $\mathrm{C} 1 \mathrm{~A}-\mathrm{C} 2 \mathrm{~A}-\mathrm{C} 3 \mathrm{~A}$ & $122.3(8)$ & $\mathrm{C} 4 \mathrm{~B}-\mathrm{C} 3 \mathrm{~B}-\mathrm{H} 3 \mathrm{~B}$ & 121.00 \\
\hline $\mathrm{C} 1 \mathrm{~B}-\mathrm{C} 2 \mathrm{~B}-\mathrm{C} 3 \mathrm{~B}$ & $121.1(8)$ & $\mathrm{C} 4 \mathrm{~A}-\mathrm{C} 5 \mathrm{~A}-\mathrm{H} 5 \mathrm{~A}$ & 120.00 \\
\hline $\mathrm{C} 2 \mathrm{~A}-\mathrm{C} 3 \mathrm{~A}-\mathrm{C} 4 \mathrm{~A}$ & $117.7(8)$ & $\mathrm{C} 6 \mathrm{~A}-\mathrm{C} 5 \mathrm{~A}-\mathrm{H} 5 \mathrm{~A}$ & 120.00 \\
\hline $\mathrm{C} 2 \mathrm{~B}-\mathrm{C} 3 \mathrm{~B}-\mathrm{C} 4 \mathrm{~B}$ & $118.8(9)$ & $\mathrm{C} 4 \mathrm{~B}-\mathrm{C} 5 \mathrm{~B}-\mathrm{H} 5 \mathrm{~B}$ & 121.00 \\
\hline $\mathrm{I} 4 \mathrm{~A}-\mathrm{C} 4 \mathrm{~A}-\mathrm{C} 3 \mathrm{~A}$ & $118.9(7)$ & $\mathrm{C} 6 \mathrm{~B}-\mathrm{C} 5 \mathrm{~B}-\mathrm{H} 5 \mathrm{~B}$ & 121.00 \\
\hline $\mathrm{I} 4 \mathrm{~A}-\mathrm{C} 4 \mathrm{~A}-\mathrm{C} 5 \mathrm{~A}$ & $119.0(7)$ & $\mathrm{C} 1 \mathrm{~A}-\mathrm{C} 6 \mathrm{~A}-\mathrm{H} 6 \mathrm{~A}$ & 120.00 \\
\hline $\mathrm{C} 3 \mathrm{~A}-\mathrm{C} 4 \mathrm{~A}-\mathrm{C} 5 \mathrm{~A}$ & $122.0(9)$ & $\mathrm{C} 5 \mathrm{~A}-\mathrm{C} 6 \mathrm{~A}-\mathrm{H} 6 \mathrm{~A}$ & 119.00 \\
\hline $\mathrm{I} 4 \mathrm{~B}-\mathrm{C} 4 \mathrm{~B}-\mathrm{C} 3 \mathrm{~B}$ & $119.1(7)$ & $\mathrm{C} 1 \mathrm{~B}-\mathrm{C} 6 \mathrm{~B}-\mathrm{H} 6 \mathrm{~B}$ & 119.00 \\
\hline $\mathrm{I} 4 \mathrm{~B}-\mathrm{C} 4 \mathrm{~B}-\mathrm{C} 5 \mathrm{~B}$ & $119.1(7)$ & $\mathrm{C} 5 \mathrm{~B}-\mathrm{C} 6 \mathrm{~B}-\mathrm{H} 6 \mathrm{~B}$ & 119.00 \\
\hline $\mathrm{C} 11-\mathrm{C} 1-\mathrm{C} 2-\mathrm{C} 1 \mathrm{~A}$ & $44.3(8)$ & $\mathrm{C} 6 \mathrm{~B}-\mathrm{C} 1 \mathrm{~B}-\mathrm{C} 2-\mathrm{C} 1 \mathrm{~A}$ & $-134.9(8)$ \\
\hline $\mathrm{C} 11-\mathrm{C} 1-\mathrm{C} 2-\mathrm{C} 1 \mathrm{~B}$ & $175.3(6)$ & $\mathrm{C} 2-\mathrm{C} 1 \mathrm{~B}-\mathrm{C} 2 \mathrm{~B}-\mathrm{C} 3 \mathrm{~B}$ & $175.7(8)$ \\
\hline $\mathrm{C} 12-\mathrm{C} 1-\mathrm{C} 2-\mathrm{C} 1 \mathrm{~A}$ & $-77.2(8)$ & $\mathrm{C} 6 \mathrm{~B}-\mathrm{C} 1 \mathrm{~B}-\mathrm{C} 2 \mathrm{~B}-\mathrm{C} 3 \mathrm{~B}$ & $-4.0(13)$ \\
\hline $\mathrm{C} 12-\mathrm{C} 1-\mathrm{C} 2-\mathrm{C} 1 \mathrm{~B}$ & $53.8(8)$ & $\mathrm{C} 2-\mathrm{C} 1 \mathrm{~B}-\mathrm{C} 6 \mathrm{~B}-\mathrm{C} 5 \mathrm{~B}$ & $-175.6(8)$ \\
\hline $\mathrm{C} 13-\mathrm{C} 1-\mathrm{C} 2-\mathrm{C} 1 \mathrm{~A}$ & $163.1(6)$ & $\mathrm{C} 2 \mathrm{~B}-\mathrm{C} 1 \mathrm{~B}-\mathrm{C} 6 \mathrm{~B}-\mathrm{C} 5 \mathrm{~B}$ & $4.0(13)$ \\
\hline $\mathrm{Cl} 3-\mathrm{C} 1-\mathrm{C} 2-\mathrm{C} 1 \mathrm{~B}$ & $-65.9(8)$ & $\mathrm{C} 1 \mathrm{~A}-\mathrm{C} 2 \mathrm{~A}-\mathrm{C} 3 \mathrm{~A}-\mathrm{C} 4 \mathrm{~A}$ & $0.9(13)$ \\
\hline $\mathrm{C} 2 \mathrm{~A}-\mathrm{C} 1 \mathrm{~A}-\mathrm{C} 2-\mathrm{C} 1$ & $-135.2(8)$ & $\mathrm{C} 1 \mathrm{~B}-\mathrm{C} 2 \mathrm{~B}-\mathrm{C} 3 \mathrm{~B}-\mathrm{C} 4 \mathrm{~B}$ & $0.7(14)$ \\
\hline $\mathrm{C} 2 \mathrm{~A}-\mathrm{C} 1 \mathrm{~A}-\mathrm{C} 2-\mathrm{C} 1 \mathrm{~B}$ & $94.9(9)$ & $\mathrm{C} 2 \mathrm{~A}-\mathrm{C} 3 \mathrm{~A}-\mathrm{C} 4 \mathrm{~A}-\mathrm{I} 4 \mathrm{~A}$ & $-177.3(6)$ \\
\hline $\mathrm{C} 6 \mathrm{~A}-\mathrm{C} 1 \mathrm{~A}-\mathrm{C} 2-\mathrm{C} 1$ & $44.5(12)$ & $\mathrm{C} 2 \mathrm{~A}-\mathrm{C} 3 \mathrm{~A}-\mathrm{C} 4 \mathrm{~A}-\mathrm{C} 5 \mathrm{~A}$ & $0.1(14)$ \\
\hline $\mathrm{C} 6 \mathrm{~A}-\mathrm{C} 1 \mathrm{~A}-\mathrm{C} 2-\mathrm{C} 1 \mathrm{~B}$ & $-85.4(10)$ & $\mathrm{C} 2 \mathrm{~B}-\mathrm{C} 3 \mathrm{~B}-\mathrm{C} 4 \mathrm{~B}-\mathrm{I} 4 \mathrm{~B}$ & $-174.4(7)$ \\
\hline $\mathrm{C} 2-\mathrm{C} 1 \mathrm{~A}-\mathrm{C} 2 \mathrm{~A}-\mathrm{C} 3 \mathrm{~A}$ & $178.4(8)$ & $\mathrm{C} 2 \mathrm{~B}-\mathrm{C} 3 \mathrm{~B}-\mathrm{C} 4 \mathrm{~B}-\mathrm{C} 5 \mathrm{~B}$ & $2.7(15)$ \\
\hline $\mathrm{C} 6 \mathrm{~A}-\mathrm{C} 1 \mathrm{~A}-\mathrm{C} 2 \mathrm{~A}-\mathrm{C} 3 \mathrm{~A}$ & $-1.4(13)$ & $\mathrm{I} 4 \mathrm{~A}-\mathrm{C} 4 \mathrm{~A}-\mathrm{C} 5 \mathrm{~A}-\mathrm{C} 6 \mathrm{~A}$ & $176.9(7)$ \\
\hline $\mathrm{C} 2-\mathrm{C} 1 \mathrm{~A}-\mathrm{C} 6 \mathrm{~A}-\mathrm{C} 5 \mathrm{~A}$ & $-178.8(9)$ & $\mathrm{C} 3 \mathrm{~A}-\mathrm{C} 4 \mathrm{~A}-\mathrm{C} 5 \mathrm{~A}-\mathrm{C} 6 \mathrm{~A}$ & $-0.5(15)$ \\
\hline $\mathrm{C} 2 \mathrm{~A}-\mathrm{C} 1 \mathrm{~A}-\mathrm{C} 6 \mathrm{~A}-\mathrm{C} 5 \mathrm{~A}$ & $1.0(14)$ & $\mathrm{I} 4 \mathrm{~B}-\mathrm{C} 4 \mathrm{~B}-\mathrm{C} 5 \mathrm{~B}-\mathrm{C} 6 \mathrm{~B}$ & $174.5(7)$ \\
\hline $\mathrm{C} 2 \mathrm{~B}-\mathrm{C} 1 \mathrm{~B}-\mathrm{C} 2-\mathrm{C} 1$ & $-86.2(10)$ & $\mathrm{C} 3 \mathrm{~B}-\mathrm{C} 4 \mathrm{~B}-\mathrm{C} 5 \mathrm{~B}-\mathrm{C} 6 \mathrm{~B}$ & $-2.7(14)$ \\
\hline $\mathrm{C} 2 \mathrm{~B}-\mathrm{C} 1 \mathrm{~B}-\mathrm{C} 2-\mathrm{C} 1 \mathrm{~A}$ & $45.5(11)$ & $\mathrm{C} 4 \mathrm{~A}-\mathrm{C} 5 \mathrm{~A}-\mathrm{C} 6 \mathrm{~A}-\mathrm{C} 1 \mathrm{~A}$ & $-0.1(15)$ \\
\hline
\end{tabular}


supporting information

$\mathrm{C} 6 \mathrm{~B}-\mathrm{C} 1 \mathrm{~B}-\mathrm{C} 2-\mathrm{C} 1 \quad 93.5(9) \quad \mathrm{C} 4 \mathrm{~B}-\mathrm{C} 5 \mathrm{~B}-\mathrm{C} 6 \mathrm{~B}-\mathrm{C} 1 \mathrm{~B} \quad-0.8(13)$

Hydrogen-bond geometry $\left(\AA,{ }^{\circ}\right)$

\begin{tabular}{lllll}
\hline$D-\mathrm{H} \cdots A$ & $D-\mathrm{H}$ & $\mathrm{H} \cdots A$ & $D \cdots A$ & $D-\mathrm{H} \cdots A$ \\
\hline $\mathrm{C} 6 A-\mathrm{H} 6 A \cdots \mathrm{Cl} 2$ & 0.93 & 2.65 & $3.335(10)$ & 131 \\
\hline
\end{tabular}

